\title{
Biomechanics and Bionics in Sport
}

\section{Editorial}

Biomechanics serves sport circles since more than a century. At the end of the 19th century famous English photographer Muybridge who was working in the United States, made thousands of serial photographs. Among them were also photographs devoted to sport. In France great scientist Ettiene-Jules Marey (physician, physiologist, biomechanist, president of the French Academy of Sciences) made several photographs of sport competitors. In the 20th century many biomechanists all over the world were working within sport biomechanics. There are such areas of interest like: body morphology, training loads, technique and tactics of movement, influence of environment, interaction of sportsperson and equipment and facilities. Body geometry, mass and moment of inertia, location of center of mass and their changes in time are main quantities investigated in sportsperson's morphology. Garments (especially shoes and boots) and accessories are also important factors influencing the final outcome. Accessories are those improving especially safety and aerodynamics of sportsperson's body. Training loads acting on the body should be optimized in order to avoid over-loading. Technique of movement is described using kinematic and dynamic quantities (displacement, time, velocity, acceleration, force, moment of force, momentum, impulse) both in transversal and rotational movements. Tactics is investigated for one sportsperson, group (formation), and the whole team and is described in kinematic quantities. Environment (air, water, snow) is important factor influencing final result. There are many kinds of sport equipment. Every discipline has its own equipment and facilities, especially balls, rackets, boats, and sport surfaces both natural and artificial.

All above mentioned problems are investigated using many kinds of biomechanical equipment. For research of body morphology mostly images are used. For kinematics data capture images and also electronic equipment, e.g. inertial measurement unit (IMU, i.e. high frequency acceleration sensors), radio equipment (radar, global navigation satellite systems), laser equipment are applied. For dynamic data force platforms, both tensometric and piezoelectric (Figure 1A), multi-sensor insoles (Figure 1B) and other equipment is used. There are different areas of using robots within sport. They are present during training (e.g. helping in providing training or as sparringpartner). They are serving as models in real sport performance, helping organizers of sport events, and other activities.

Bionics as biologically inspired engineering (BIE) appeared about 100 years ago (1907) when the first organic material was synthetized, namely bakelite. The term bionics was coined by Jack E. Steele in 1958. In the 20th century BIE developed enormously. It exists for example in the name of Wyss Institute for Biologically Inspired Engineering at Harvard University (Cambridge, Mass.). Some people explain the word bionics as combination of words biology and electronics. Some other scientists and engineers talking on biologically inspired materials and devices use a word biomimetics.

Bionics in sports exists especially in hydro- and aeronautics. All boats have elongated shape in order to have small water drag like almost all fishes have. Also shapes of flying objects are elongated. In addition wings have cross-section similar to those of birds. But in one case sport federation resigned from bionic shape. This is shark skin-
Volume 2 Issue 3 - 2018

Wlodzimierz S Erdmann

Department of Biomechanics and Sport Engineering, Poland

Correspondence: Department of Biomechanics and Sport Engineering, J. Sniadecki University of Physical Education and Sport, Gdansk, Poland, Email:werd@awf.gda.pl

Received: May 15, 2018 | Published: May 17, 2018

like uniforms used by swimmers. The costume is expensive and also federation wants that human fitness must be present in water and not engineering.

Recently the most spectacular presence of bionics is in sport for disabled people. In 2016 Zurich Federal Polytechnic organized Cybathlon, i.e. competition where sophisticated engineering helped disabled people in six disciplines: 1) powered arm prosthesis race, 2) powered leg prosthesis race, 3 ) powered exoskeleton race, 4) powered wheelchair race, 5) functional electrical stimulation bike race, 6) brain-computer interface race. Competitors had to overcome several obstacles on the track (Figure 2), except for the two last disciplines.

A

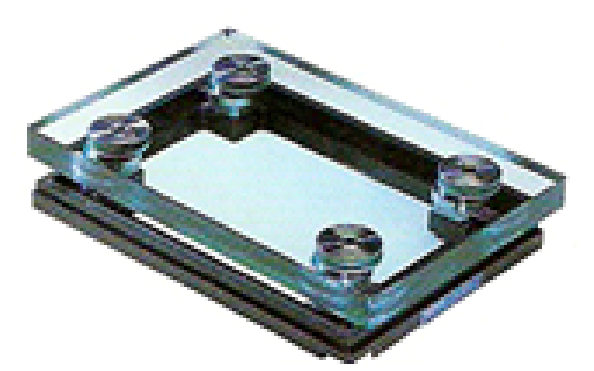

B

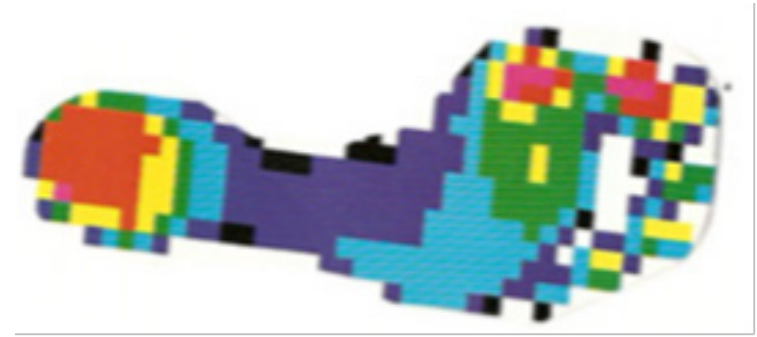

Figure I Measuring equipment for dynamic data capture: A-force platform (Kistler) for measurement e.g. of take-off in jumps, B-multisensor insole (Emed) for measurement of distribution of load acting on foot

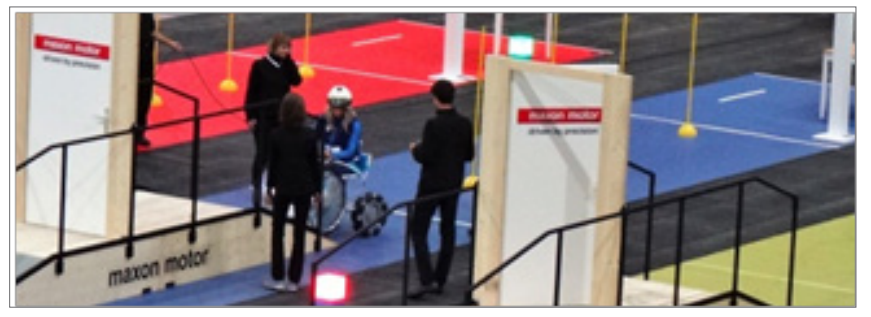

Figure 2 Track with obstacles during Cybathlon 2016 at ETH Zurich. 
MedCrave Online Journal of Applied Bionics and Biomechanics (MOJABB) is an International, Peer reviewed Journal of advanced technological developments based on the science of biological systems. The journal solicits research papers for its forthcoming issue in all aspects of bionic science and engineering including fundamental understandings of animals and plants for bionic engineering, such as locomotion and behaviors of animals, structures, composites, morphology and physical properties of plants and natural materials, applications of such understandings in engineering, technology and designs. Journal accepts Research Papers, Review Articles, Short Communications, Case Reports, Mini-Reviews, Opinions, Letter to Editors, etc. in this field which will be enlightening the scientific community. On behalf of the editorial team of the journal I invite authors from all over the world to share their innovative research and findings. Further I assure that the contents submitted to MedCrave will enjoy more visibility and will be peer reviewed too. You can submit your works on any topic of your expertise within the scope of journal. Journal ensures to share only high-quality content, so there is no room for copied or doubtful content to be published. You can submit work on any topic relevant to science. The content should be unique, original and the presentation must be of potential interest to the readers. You can submit your research articles too. These research papers must be original and must be in the major field of science. The research articles can include the findings and the methodology you used. You can also compile your evidences that lead to your conclusions. So once again I beseech timely submission of research papers for prospective publication in upcoming issue of the journal.

\section{Acknowledgments}

None.

\section{Conflict of interest}

Authors declare that there is no conflict of interest. 